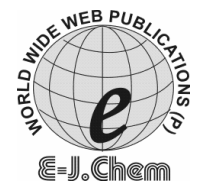

\title{
Synthesis, Characterization and Application of Coatings Based on Epoxy Novolac and Liquid Rubber Blend
}

\author{
HEMANT KUMAR*, S.K TRIPATHI, SUKHEN MISTRY ${ }^{\S}$ \\ and GANESHDATT BAJPAI ${ }^{\S}$ \\ Department of Chemistry, \\ Vikramajit Singh Sanatan Dharma College (V.S.S.D), \\ ${ }^{\S}$ Department of Oil \& Paint Technology, \\ Harcourt Butler Technological Institute (HBTI), \\ Nawabganj, Kanpur-208 002, India. \\ hemantkumar.phd2007@gmail.com
}

Received 9 November 2008; Accepted 16 January 2009

\begin{abstract}
Phenol-based novolac resins were synthesized with different mole ratios of phenol-to-formaldehyde. These novolac resins were epoxidized with molar excess of epichlorohydrin at $120{ }^{\circ} \mathrm{C}$ in basic medium. Novolac and epoxy novolac resin were characterized by FTIR, NMR and GPC analysis. Molecular weight was found to be 838 . The epoxidized novolac resins were separately blended with different weight ratios of carboxyl-terminated polybutadiene liquid rubber ranging between $0-25$ wt $\%$ with an interval of $5 \mathrm{wt} \%$. All the blends were cured at $150{ }^{\circ} \mathrm{C}$ with $40 \mathrm{wt} \%$ polyamide. The cured films of blend samples were checked for use them in coating applications.
\end{abstract}

Keywords: Epoxidized novolac, Liquid rubber blend, Epoxy novolac, Synthesis.

\section{Introduction}

Epoxy resins are widely used as high-performance protective coatings, structural adhesives, low-stress IC encapsulants and matrix resins for composites. When cured these resins are highly cross-linked and become amorphous thermosets. They are brittle and have poor resistance to crack growth. They are highly employed in "joining and fastening technology" in many industries. The epoxy resin network is formed during the cross-linking reaction using a wide variety of cross-linking agents or hardeners such as acids ${ }^{1}$, anhydrides ${ }^{2}$, and amines ${ }^{3}$. The reaction leads to the formation of a three dimensional system which is found to be insoluble in usual solvents. Toughening of epoxy resins with low-molecular weight liquid rubbers has been studied ${ }^{4-8}$. Many authors ${ }^{9-11}$ have also made comments on the increasingly 
wide use of rubber modified epoxy resins as structural adhesives and as the matrix for fiber composites. Because of their properties, epoxy resins have many commercial applications. For this reason, it was deemed interesting to modify them with rubber particles. Rubber was added to the uncured epoxy resins and after the cross-linking reactions the rubber-modified epoxy resins exhibited a two-phase microstructure consisting of relatively small rubber particles dispersed in a matrix of epoxy. Toughening of epoxy resin is extremely useful because application of this polymeric material imparts resistance against mechanical deformation at different loading rates. The brittle nature of the epoxy is the result of catastrophic strain localization in the form of crazes, which may cause premature fracture at a relatively small macroscopic deformation ${ }^{12}$. Rubber toughening ${ }^{13}$ or cross-linking ${ }^{14}$ of epoxy system results in the increase in hardening modulus. Therefore, we have tried to produce such modified epoxy matrices by physical blending of phenol based epoxy novolac resin with CTPB and studied the effect of CTPB addition in the physical and chemical resistance properties of the coating films.

\section{Experimental}

Phenol (LR), formaldehyde (40\% solution), oxalic acid, sodium hydroxide, epichlorohydrin, hydroquinone (All from M/s Thomas Baker Chemicals Ltd., Mumbai), polyamide (M/s Resinova Chemie Ltd., Kanpur) with amine value $240-400 \mathrm{mg} \mathrm{KOH} / \mathrm{g}$, and carboxyl terminated polybutadiene (CTPB)(Hycar 2000x162) as gift sample from M/s Emerald Performance Materials, LLC, Hong Kong were used during the investigation.

\section{Preparation of phenol based epoxidized novolac resin}

Phenol based novolac resin with mole ratio 1:0.6 of o- phenol (P) to formaldehyde (F) was prepared using oxalic acid as catalyst by a method similar to that adopted in previous article ${ }^{15}$. The initial $\mathrm{pH}$ of the reaction mixture was 6.0 , which reduced to a value of 4.8 after $5 \mathrm{~h}$ of reaction at $120{ }^{\circ} \mathrm{C}$. Free-formaldehyde content was checked after every $45 \mathrm{~min}$ to check the progress of the methylolation reaction ${ }^{16}$. These phenol based novolac- type phenolic resins were epoxidized by a method similar to the method given in literature ${ }^{17}$ with epichlorohydrin in basic medium for a period of $5 \mathrm{~h}$ at $120^{\circ} \mathrm{C}$. The resulting viscous product was stored for further analysis. All the samples are designated according to Table 1.

Table 1. Variation of cure time for the blend samples cured with polyamide at $150{ }^{\circ} \mathrm{C}$.

\begin{tabular}{ccccccc}
\hline $\begin{array}{c}\text { S. } \\
\text { No. }\end{array}$ & $\begin{array}{c}\text { Sample } \\
\text { code }^{\mathrm{a}}\end{array}$ & $\begin{array}{c}\text { EN resin, } \\
\mathrm{wt} \%\end{array}$ & $\begin{array}{c}\mathrm{CTPB}, \\
\mathrm{wt} \%\end{array}$ & $\begin{array}{c}\mathrm{T}_{\mathrm{p}}^{\mathrm{b}}, \\
{ }^{\circ} \mathrm{C}\end{array}$ & $\begin{array}{c}{ }_{\mathrm{t}} \text { cure, } \\
\mathrm{min}\end{array}$ & $\begin{array}{c}{ }_{\mathrm{t}} \text { cure } \\
\mathrm{min}\end{array}$ \\
\hline 1. & $\mathrm{PNC}_{0}$ & 100 & 0 & 165 & 395 & 370 \\
2. & $\mathrm{PNC}_{5}$ & 95 & 5 & 150 & 350 & 340 \\
3. & $\mathrm{PNC}_{10}$ & 90 & 10 & 145 & 330 & 320 \\
4. & $\mathrm{PNC}_{15}$ & 85 & 15 & 147 & 320 & 300 \\
5. & $\mathrm{PNC}_{20}$ & 80 & 20 & 150 & 325 & 315 \\
6. & $\mathrm{PNC}_{25}$ & 75 & 25 & 155 & 335 & 320 \\
\hline
\end{tabular}

EN: Phenol-bases epoxidized novolac resin; ${ }^{a} E$ : Epoxy; C: CTPB; The first numeric digit is the first digit from the weight percent of epoxy resin and the second numeric digit is from the first digit of weight percentage of CTPB.

${ }^{b}$ Results from the dynamic DSC scans. $T_{p}$ : Maximum temperature of exothermic peak. ${ }^{c}$ cure time without curing agent; ${ }^{d}$ cure time with polyamide. 


\section{Analytical methods}

Characterizations of blend samples of uncured and cured samples were recorded on a PerkinElmer (Model 843) Infra-red Spectrophotometer, using $\mathrm{KBr}$ pellet. Dynamic scans were recorded with a Perkin-Elmer differential scanning calorimeter (DSC) (Model Dymaond DSC; Switzerland) at a heating rate of $10{ }^{\circ} \mathrm{C} / \mathrm{min}$ in inert atmosphere up to a temperature of $170{ }^{\circ} \mathrm{C}$ to get the cure temperature. Gel permeation chromatograph was recorded with a Shimadzu Europa GmbH, Duisburg, Germany high performance GPC (Model LC-6A).

\section{Preparation of coating films}

The curing of blend samples with $40 \mathrm{wt} \%$ polyamide were performed in an air oven $(\mathrm{M} / \mathrm{s}$ Indian Equipment Corporation, Mumbai, India) at $140{ }^{\circ} \mathrm{C}$. The panels were prepared by applying the blend samples on sandblasted steel sheet panels of sized $150 \times 100 \times 1.25 \mathrm{~mm}$ with a Bird Film Applicator (Sheen, UK). These panels were sealed on three sides with molten paraffin wax. A dry film thickness of about $100 \mu \mathrm{m}$ was maintained on all the panels.

\section{Curing of blend samples}

The curing of blend samples with $40 \mathrm{wt} \%$ polyamide was performed in an air oven $(\mathrm{M} / \mathrm{s}$ Indian Equipment Corporation, Mumbai, India) at $140{ }^{\circ} \mathrm{C}$. These films were cured at $150{ }^{\circ} \mathrm{C}$ and then various mechanical and chemical resistance properties of the films were evaluated.

\section{Characterization of coating Films}

The cure films were tested for their different optical, mechanical and chemical resistance properties as per test methods viz. gloss at $60^{\circ}$ (ASTM:D 523-99), scratch hardness (ASTM:D 5178), pPNCil hardness (ASTM:D 3363-00), chemical resistance (ASTM: D 1308) and solvent resistance (ASTM : D 5402).

The adhesion and flexibility of the cured films of the resin was tested by putting the prepared panels into $1 / 4$-inch mandrel keeping the coated side downward. Then the two plates, connected to the mandrel, were readily bent. The bent portion of the panels was examined for any damage in the film. The impact resistance of the cured film samples was conducted by dropping a hemi-spherical shaped four-pound weight from 40 inch height over the panels. The tests were carried out with the uncoated side of the panel facing the falling weights. The panels were also examined for a visible change in the conditions of the film samples at regular intervals when immersed in different chemicals like solvents, acids, and alkalies at ambient temperature for the period of twelve months.

\section{Results and Discussion}

\section{FTIR-spectrum of phenol - formaldehyde novolac}

Figure 1 shows the infra-red spectra of novolac samples PN1. It is evident from the figure that, there is a band in the region of $3000-3620 \mathrm{~cm}^{-1}$ which might be due to phenolic methylol hydroxyl $(-\mathrm{OH})$ vibration present in the novolac resin. A band in the region of 1315-1409 $\mathrm{cm}^{-1}$ might be due to the presence of hydroxyl groups, (methylol hydroxyls) in the molecules. The band located at $1500 \mathrm{~cm}^{-1}$ might be assigned to phenolic ring substituted at the ortho-, parapositions. The same could be further confirmed by the appearance of absorption band at 820 $\mathrm{cm}^{-1}$ which clearly indicates the presence of aromatic ethylene bonds $(\mathrm{C}=\mathrm{C})$ of phenolic ring. A peak near $1200 \mathrm{~cm}^{-1}$ is also appearing in the spectrum is due to stretching vibration of phenol-O group. The peak appearance in the IR spectra confirms the formation of novolacs with ortho-and para-substituted phenolic rings connected via methylene linkages. 


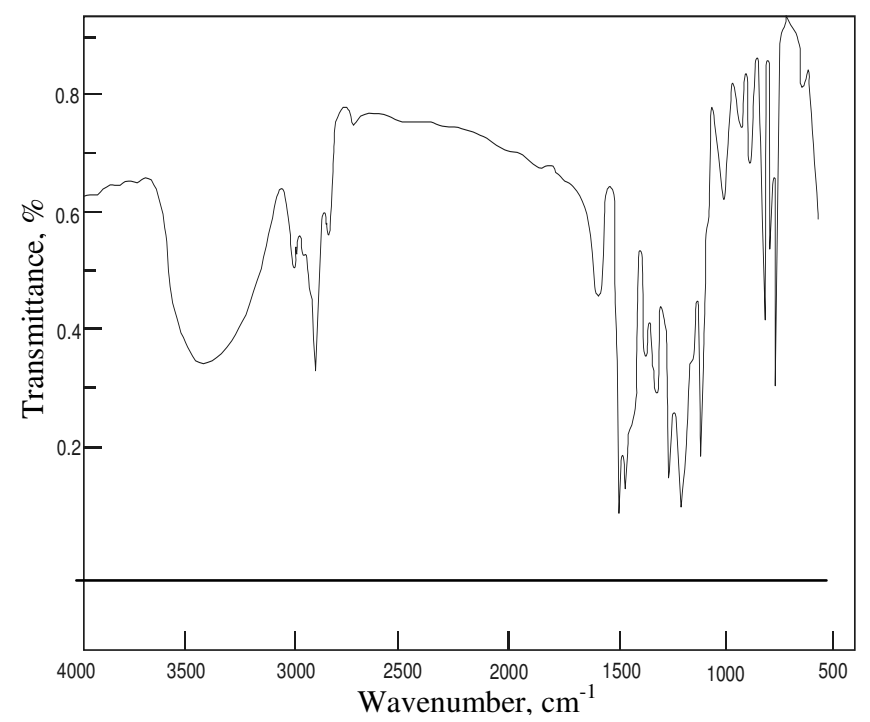

Figure 1. FTIR-spectrum of Phenol - formaldehyde novolac.

\section{${ }^{1} H$-NMR spectra of phenol - formaldehyde novolac}

Figure 2 shows the ${ }^{1} \mathrm{H}$ NMR spectra of novolac. A multiplet observed in the range of 3.4$4.2 \mathrm{ppm}$ might be due to aliphatic protons present in the structure of the novolac resin. The appearance of multiplet near 6.4-7.4 ppm might be due to the aromatic protons present in the structure. There also appears another multiplet in the region of 9.0-9.6 ppm, which could be assigned to the hydroxyl group functionality of the novolac resins. The integrated area of the multiplites is taken as a measure of aliphatic, aromatic and hydroxyl proton units in the structure.

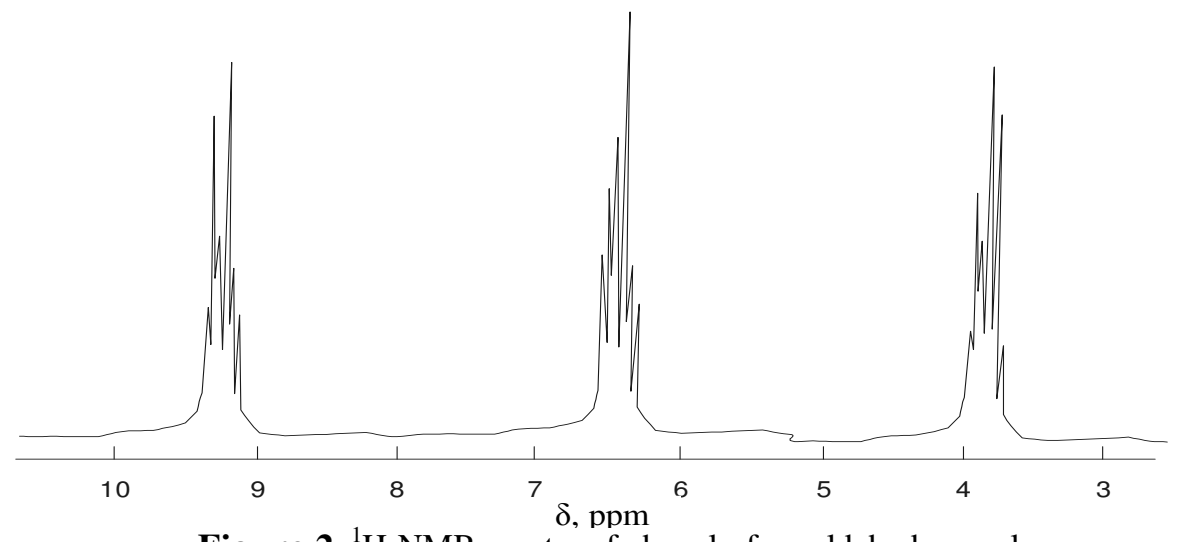

Figure 2. ${ }^{1} \mathrm{H}-\mathrm{NMR}$ spectra of phenol - formaldehyde novolac.

\section{FTIR spectra of epoxidised novolac}

Figure 3 shows the FTIR-spectra of epoxidised novolacs. It is evident from the figure that, there is a broad band near $3200-3650 \mathrm{~cm}^{-1}$ which can be attributed to the hydroxyl stretching vibration of phenolic group. The absorption band near $2890 \mathrm{~cm}^{-1}$ might be due to $\mathrm{CH}_{2}$ vibrations. The absorption bands near 1520,1590 and $1620 \mathrm{~cm}^{-1}$, might be due to vibration of 
substituted aromatics. The absorption band at $1255-1268 \mathrm{~cm}^{-1}$ indicates the presence of oxirane group of epoxy. The absorption bands near 920 and $880 \mathrm{~cm}^{-1}$ appear due to the ring deformation of terminal epoxy group in each spectrum, which confirms the presence of epoxy groups in this polymer. The regions of 750-790, 820-850 and $910-920 \mathrm{~cm}^{-1}$ might be due to $o-, p$ - and ring-substitution of phenolic ring respectively.

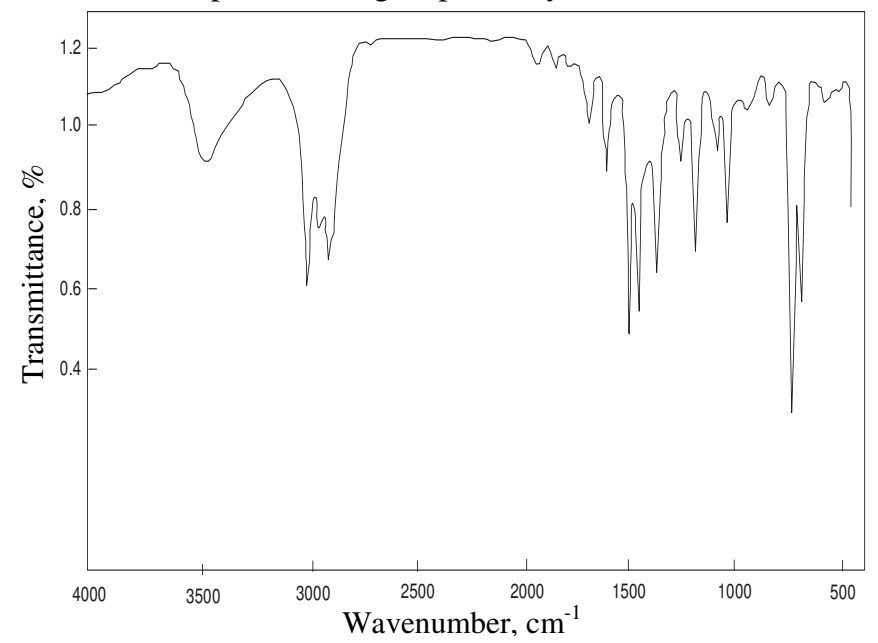

Figure 3. FTIR spectra of epoxidised novolac.

\section{${ }^{1} H$-NMR spectra of epoxidized novolac}

It is clear from Figure 4 that, there is a multiplet in the region of 2.38-2.90 ppm which might be due to presence of aliphatic protons attached to the $>\mathrm{CH}_{2}$ group of the epoxy ring. A broad singlet appeared in Figure 4 near 3.08-3.33 ppm which might correspond to the aliphatic protons attached to $>\mathrm{CH}$ group of the epoxy ring. Appearance of multiplet in the region between 3.39 and $3.43 \mathrm{ppm}$ corresponds to the overlapping of the aliphatic protons due to $>\mathrm{CH}_{2}$ group attached to epoxy ring on one side and oxygen $(\mathrm{O})$ to the other side, and due to $>\mathrm{CH}_{2}$ attached to the aromatic ring on both the sides. A multiplet, in the region between 6.64 and $7.45 \mathrm{ppm}$ might be due to the aromatic protons of benzene nucleus.

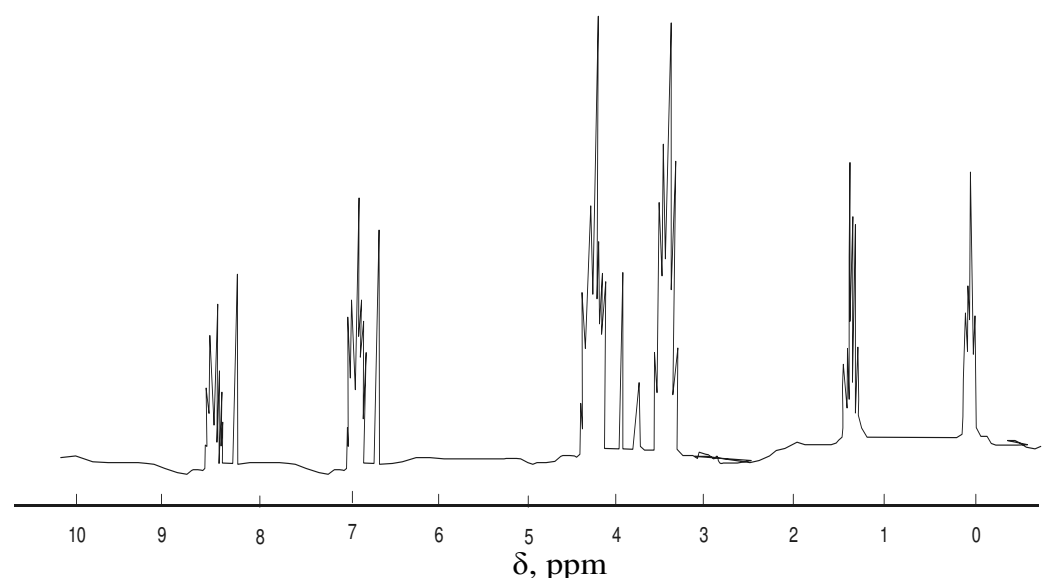

Figure 4. 1H-NMR spectra of epoxidized novolac. 


\section{Mechanical properties of coating films}

Table 2 showed the surface and mechanical properties of cured films of blend samples. The table clearly indicated that the cured films of all blend samples containing 5-25 wt\% CTPB showed smooth and uniform with semi-glossy surfaces. All the films of blend samples passed through adhesion and flexibility test except for pure epoxy samples, viz., sample EN20 and EN30. The blend samples showed improved impact resistance than that of pure epoxy resin. The flexibility improvement may be thought due to the presence of some dissolved rubber particles inside the epoxy matrix. The decrease of crosslink density might also be an indicative of improved flexibility whereas cavitations of rubber particles inside the epoxy matrix improved the impact resistance of the films of blend samples. These properties illustrated that the coatings prepared from the blend samples had a quality appearance, indicating homogeneity throughout the coatings and relatively good film properties.

Table 2. Mechanical properties of modified epoxy and CTPB blend films.

\begin{tabular}{clcccccc}
\hline \multirow{2}{*}{ S.No. } & \multicolumn{1}{c}{ Property } & $\mathrm{PNC}_{0}$ & $\mathrm{PNC}_{5}$ & $\mathrm{PNC}_{10}$ & $\mathrm{PNC}_{15}$ & $\mathrm{PNC}_{20}$ & $\mathrm{PNC}_{25}$ \\
\hline 1. & Scratch Hardness, g & 2100 & 2400 & 2600 & 2700 & 2800 & 3000 \\
2. & PPNCil Hardness & $2 \mathrm{H}$ & $2 \mathrm{H}$ & $3 \mathrm{H}$ & $3 \mathrm{H}$ & $3 \mathrm{H}$ & $4 \mathrm{H}$ \\
3. & Cross-Hatch Hardness, $\%$ & $100 \%$ & $100 \%$ & $100 \%$ & $100 \%$ & $100 \%$ & $100 \%$ \\
4. & Flexibility test in 1/4" mandrel & Pass & Pass & Pass & Pass & Pass & Pass \\
5. & Impact resistance, cm & 90 & 105 & 115 & 135 & 140 & 150 \\
6. & Gloss at $60^{\circ}, \%$ & 70 & 70 & 75 & 80 & 81 & 85 \\
\hline
\end{tabular}

Chemical resistance properties of blend samples

Table 3 shows the comparative acids and alkalis resistance of the cured films of phenolbased epoxidized novolac resin and its blends with different weight ratio of CTPB. A quick perusal of Table 3 clearly illustrated that the film of coatings prepared from $\mathrm{PNC}_{15}$ epoxy have offered maximum resistance towards different concentrations of acids and alkalis as compared to the films of other epoxy blends.

Table 3. Comparative study of acid, alkali and solvent resistance of modified epoxy and CTPB blend films ${ }^{\mathrm{a}}$ cured at $150{ }^{\circ} \mathrm{C}$ showing the period after first effect observed.

\begin{tabular}{llcccccc}
\hline S.No. & \multicolumn{6}{c}{ Reagents } & \multicolumn{7}{c}{ Periods in months } \\
\cline { 3 - 7 } & & $\mathrm{PNC}_{0}$ & $\mathrm{PNC}_{5}$ & $\mathrm{PNC}_{10}$ & $\mathrm{PNC}_{15}$ & $\mathrm{PNC}_{20}$ & $\mathrm{PNC}_{25}$ \\
\hline 1. & Sulphuric acid 10\% & 6 & 8 & 10 & 11 & 10 & 10 \\
2. & Hydrochloric acid 10\% & 9 & 11 & 10 & 11 & 10 & 10 \\
3. & Acetic acid 10\% & 11 & 11 & 11 & 12 & 12 & 12 \\
4. & Sodium hydroxide 10\% & 10 & 11 & 11 & 12 & 12 & 10 \\
5. & Ammonium hydroxide 10\% & 10 & 11 & 12 & 12 & 12 & 12 \\
6. & Xylene & 10 & 11 & 11 & 12 & 12 & 12 \\
7. & Ethanol & 7 & 9 & 11 & 12 & 11 & 11 \\
8. & MEK & 5 & 8 & 10 & 12 & 9 & 9 \\
\hline
\end{tabular}

${ }^{a}$ Film thickness of about $100 \mu \mathrm{m}$ on glass panels with wax sealing from all sides.

This behavior might be attributed due to greater reactivity resulting in a more complex structure to provide these resistances as compared to other compositions. The cured films of all blend samples were completely unaffected by $10 \%$ sulphuric acid solution for the entire period of exposure of twelve months. The films when exposed to $20 \%$ sulphuric acid and 
hydrochloric acid solutions were found to be resistant for ten months whereas in other solutions, they were resistant for less than nine months. The film of blend samples containing $15 \mathrm{wt} \%$ CTPB showed better resistance towards all acid solutions as compared to the film of blend samples containing 5, 10, 20, and $25 \mathrm{wt} \%$ CTPB Table 3. The lower concentration of alkali solution less affected the film surface than higher alkali solutions Table 3. This behavior might be attributed to the fact that the formation of more crosslink structures by the addition of epoxy resin.

\section{References}

1. Bradleg T F, U S Patent, 1950, 449, 2500.

2. Caston P, U S Patent, 1943, 483, 2324.

3. Newey H A, U S Patent, 1958, 775, 2264.

4. Siebert A R, Rubber-modified thermoset resins, Advances in Chemistry Series, Washington DC, 1984, 208, 179.

5. Manzione L T, Gillham J K and Mc Pherson C A, J Appl Polym Sci., 1981, 26(889), 907.

6. Kunz S C, Sayre J A and Assink R A, Polymer, 1982, 23, 1897 and references cited therein.

7. Riew C K and Gillham J K, Editors, Advances in Chemistry Series, Washington DC, 1984, 208, 101.

8. Riew C K, Editor, Advances in Chemistry Series, 1984, 222.

9. Paul N C, Richards D H and Thompson D, Polymer, 1977, 18, 945.

10. Baucer R S, Epoxy Resin Chemistry II, ACS Symposium Series No. 221 Washington, DC, 1983.

11. Bascom W D, Cottingham R L, Tones R L and Peyser P, J Appl Polym Sci., 1979, 19, 2545.

12. Smith R J M, Brekelmans W A M and Meijer H E H, J Mater Sci., 2000, 35, 2855.

13. Smith R J M, Brekelmans W A M and Meijer H E H, J Mater Sci., 2000, 35, 2881.

14. Henkee C S and Kramer E J, J Polym Sci Polym Chem Ed., 1984, 22, 721.

15. Knop A and Schieb W, Chemistry and Application of Phenolic Resins, Springer Verlag, New York, 1979.

16. Bascom W D, Cottington R L, Jones R L and Peyser P, J Appl Polym Sci., 1975, 19, 2545.

17. Nigam V, Setua D K and Mathur G N, IUPAC Pub. II, 1998, 757. 


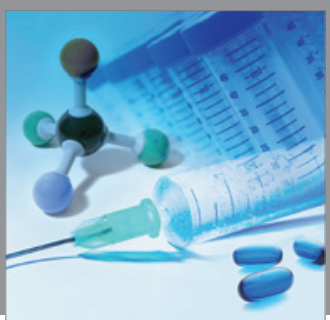

International Journal of

Medicinal Chemistry

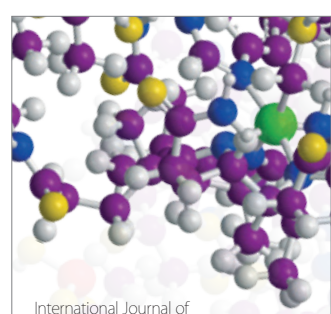

Carbohydrate Chemistry

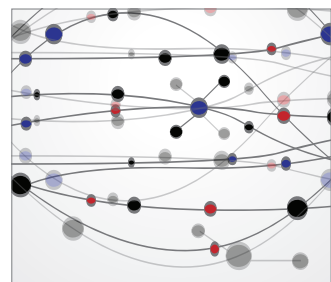

The Scientific World Journal
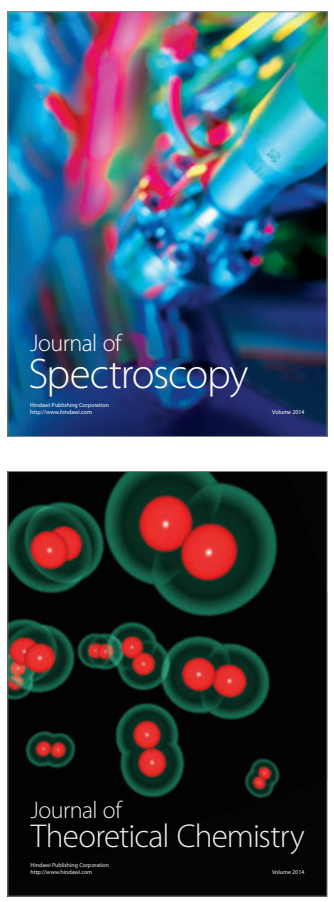
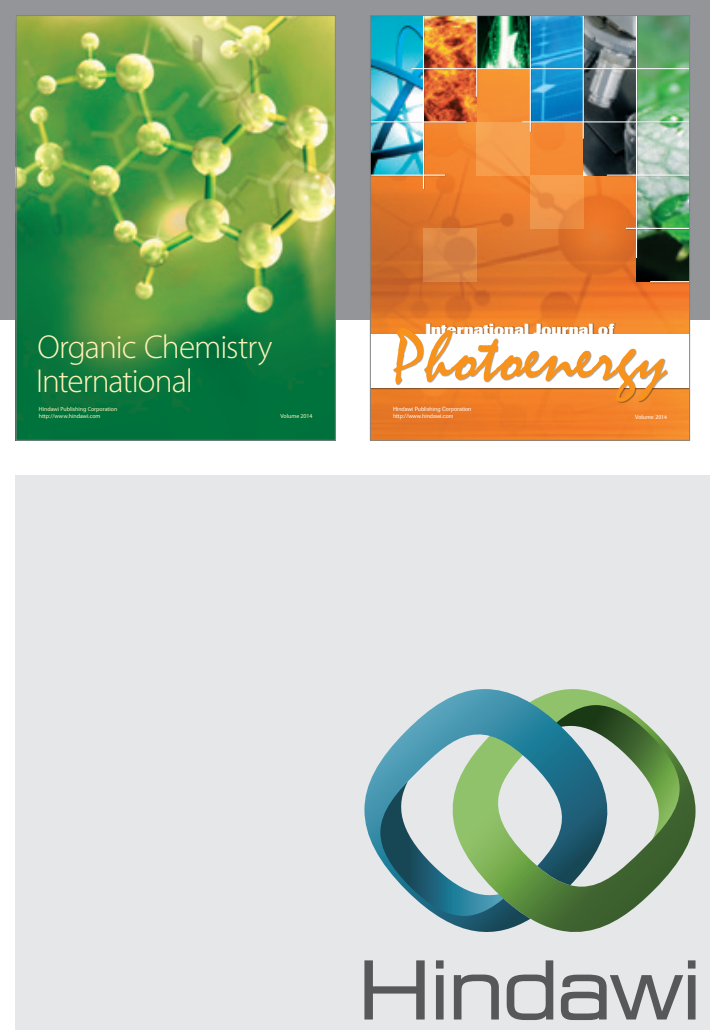

Submit your manuscripts at

http://www.hindawi.com
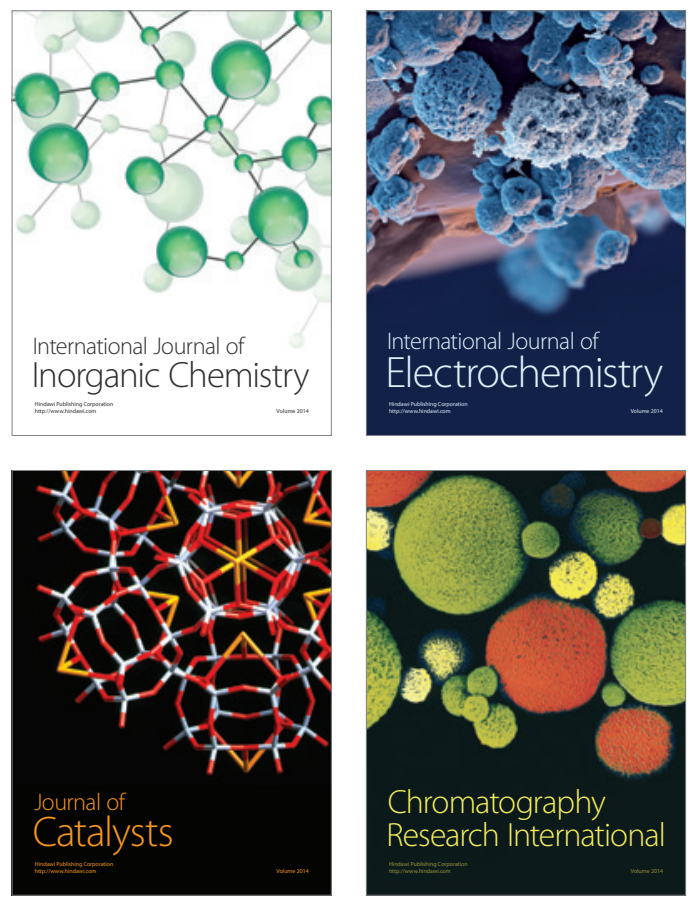
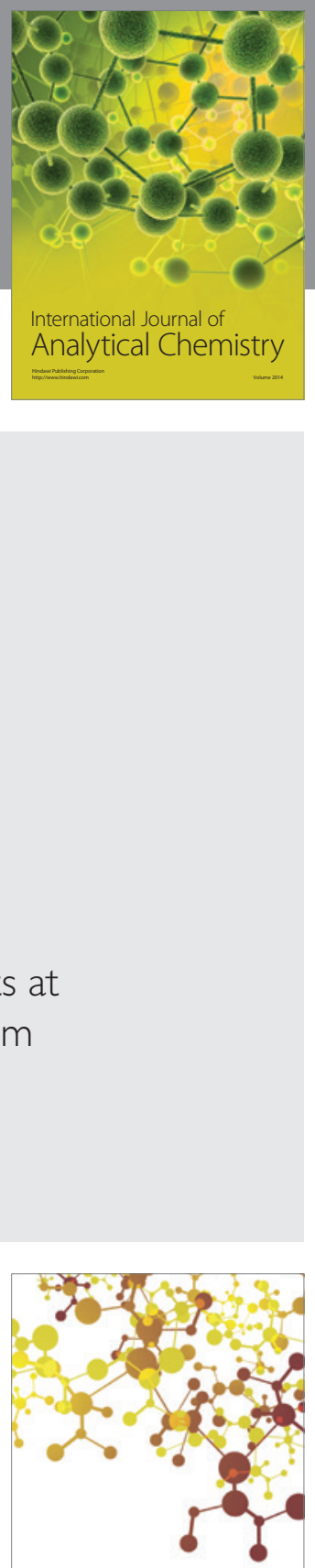

Journal of

Applied Chemistry
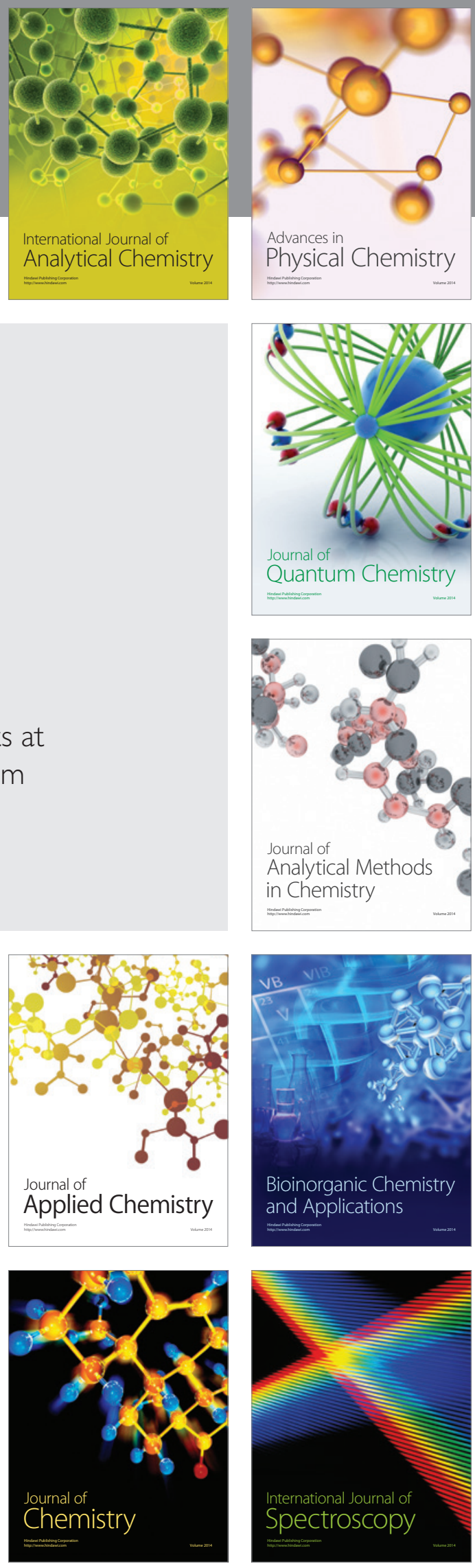